\title{
Impact of Targeted Cancer Nutrition on Hormonal Cancers
}

\section{Rachna Chhachhi*}

Nutritional Therapist, Holistic Cancer Coach, India

*Corresponding Author: Rachna Chhachhi, Nutritional Therapist, Holistic Cancer Coach, India.

Received: September 19, 2019; Published: October 10, 2019

DOI: 10.31080/ASNH.2019.03.0492

\section{Background}

Hormonal cancers like breast cancer, ovarian cancer and prostate cancer are becoming increasingly common in men and women. While prostate cancer is more common in men over the age of 50 , breast cancer has started becoming common much younger women, from the age of 27 till postmenopausal age. Ovarian cancer is more common in women in their mid-30s to late 40 s. Prostate cancer is a slow-growing cancer and often does not need aggressive medical treatment to arrest, however, most breast and ovarian cancer cases are listed and extremely aggressive.

\section{Aim of Work}

To establish the role of nutrition in cancer healing.

\section{Methodology}

In this retrospective study, patient data from 6 patients suffering from hormonal cancers like breast, ovarian and prostate cancer was collected. These patients had been under conventional treatments as well as nutritional healing to strengthen the immune system and reduce side effects of chemotherapy, radiation and surgery. Reports before, during and after chemotherapy showed quicker recovery process in breast cancer patients.

\section{Results}

In 4 prostate cancer patients, aged between 60-75 years, treated with nutritional healing, 3 patients who had not continued with medical treatments responded better to complete recovery than those who had been treated with chemotherapy and surgery.

The oldest patient responded best to nutritional healing. Some stats below.

- $\quad$ Patient was diagnosed with stage III grade 4 prostate cancer suspected metastatis to liver at the age of 75

- $\quad$ PSA was 135

- Prostate size was $46 \mathrm{cc}$

- 38 cycles of radiation were prescribed. Within 8 sittings, patient was in pain, unable to pass stool and constipated. Radiation had to be abandoned. Nutritional healing began.

- PSA came down to 19 within 3 months with nutritional healing.
- Prostate size came down to $22 \mathrm{cc}$

- At age 80, 5 years after diagnosis, reports continue to be negative for prostate cancer and quality of life is high.

- His prostate size is $14 \mathrm{cc}$ (below normal), his PSA is below normal (0.05), his inflammation markers are in range and his testosterone levels are below normal. He is on zero medication, however, he is on specific cancer nutrition treatment including supplementation for the last 5 years.

- However, he faces some side effects of radiation till date.

In another patient, 63 years, results with nutritional healing were as below

- Fatigue: drastically reduced levels

- $\quad$ PSA (>100): came down to 0.264 from March to Aug 2019

- $\quad$ Enlarged prostate, spread to lymph nodes (46 cc): came down to 29cc and now to $20 \mathrm{cc}$ (March - Aug 2019)

- High ESR (40): came down now to 12

- $\quad$ Low Vit D: is now steady at 49.2

- High uric acid (9.94): has reduced to 6.2

- $\quad$ High HbA1C (6.1): now reduced to 5.5

- Enlarged fatty liver: is still present but has reduced

In one stage III BRCA1 positive ovarian cancer case, patient came after surgery and was informed by oncologist at Sloan-Kettering that chances of recurrence were $90 \%$. She started nutritional healing, and 6 years later, is still free from cancer due to nutrition and lifestyle changes.

In one breast cancer case, stage III, BRCA 1 positive, recovery of the patient aged 28 years was much faster and side effects of chemotherapy. When chemotherapy cycles were completed, patient complained of anal fissures, blood in stool, symptoms of digestive discomfort like bloating, acidity, constipation accompanied with fatigue. All these were reversed within a period of two months after chemotherapy was over and nutritional healing continues. 


\section{Conclusion}

Even though Prostate cancer is a slow-growing cancer and often does not need aggressive medical treatment to arrest, and most breast and ovarian cancer cases are listed and extremely aggressive, results of nutritional healing accompanying medical treatments or without them achieved much higher recovery and better quality of life along with reduced chances of recurrence in BOTH male and female patients irrespective of whether the cancer was slow-growing or aggressive. Hence, whether medical treatment for cancer in any form is given or not, impact of nutrition in recovery and reduction of recurrence of cancer in the case of hormonal cancers is more successful. Patients who adapted well to nutritional healing also reported a decreased muscle wasting, decreased fatigue, near normal digestion, higher quality of life in the form of higher energy, reduced chances of recurrence and a more active life.

Volume 3 Issue 11 November 2019

(C) All rights are reserved by Rachna Chhachhi. 